\title{
Resultado perinatal na macrossomia fetal
}

\section{Perinatal results in fetal macrosomia}

\author{
Lilian Renata Fiorelli', Marcelo Zugaib²
}

\begin{abstract}
Fiorelli LR, Zugaib M. Resultado perinatal na macrossomia fetal. Rev Med (São Paulo). 2007 jul.-set.;86(3):144-7.

RESUMO: Introdução: A gestação de fetos macrossômicos aumenta o risco complicações perinatais. Método: Análise retrospectiva de 306 partos ocorridos no HU/USP entre 2002 e 2004, cujos recém-nascidos apresentaram peso $=4000$ gramas. Resultados: $A$ idade das gestantes foi de 14 a 43 anos (27,4 $\pm 6,0$ anos) e 33,9\% com paridade $>2$ partos. A idade gestacional variou de 36 a 43 semanas $(40,1 \pm 1,1)$ sendo $54,2 \%$ gestações pós-data, e o peso ao nascimento variou de 4000 a 5085 gramas $(4195,5 \pm 188,9)$. O tipo de parto foi vaginal em 141 (46,1\%) gestantes e cesárea em 165 (53,9\%) gestantes. As principais intercorrências materno-fetais foram mecôneo $(4,9 \%)$, traumatismo no recém-nascido $(2,3 \%)$, laceração vaginal $(1,9 \%)$, atonia uterina $(1,6 \%)$ e distocia do bisacromial $(1,6 \%)$. Não houve diferença significativa na ocorrência de intercorrências $(O R=0,95 ; I C=0,52-1,74)$ entre partos cesáreo e vaginal, e entre recém-nascidos com peso < ou > a 4500 gramas $(O R=1,09$; IC = 0,38-3,10). Conclusão: Identificou-se o pós-datismo, a multiparidade e a idade materna avançada relacionados a macrossomia. Esta também associa-se a cesárea, a trauma de parto e a morbidade perinatal, porém, não demostrou-se a correlação entre a via de parto ou peso de nascimento $<$ ou > de 4500gramas e a freqüência de intercorrências materno-fetais.
\end{abstract}

DESCRITORES: Macrossomia fetal. Peso ao nascer. Complicações na gravidez. Fatores de risco.

\section{INTRODUÇÃO}

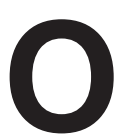

processo do crescimento e desenvolvimento fetal envolve a mãe, a placenta e o feto ${ }^{6}$ e depende do aporte de nutrientes como glicose, lipídios e aminoácidos $^{17}$. Fatores genéticos, somados ao estado materno-fetal, também influenciam no desenvolvimento ${ }^{8,17}$. Achados epidemiológicos, clínicos e experimentais indicam que o diabetes mellitus gestacional ou pré gestacional, assim como a obesidade ou excesso de ganho de peso durante a gravidez, são fatores de risco significantes para a supernutrição fetal e a macrossomia ${ }^{5,8,17,23}$. Outros fatores de risco para macrossomia fetal são multiparidade, idade materna avançada, antecedente de feto macrossômico, feto masculino e pós-datismo ${ }^{5,23,26}$.

A prevalência mundial de recém-nascidos

Prêmio Oswaldo Cruz - Área Clínica. Trabalho realizado no Hospital Universitário da Universidade de São Paulo (HU/ USP).

1. Acadêmica do $6^{\circ}$ ano de Medicina da FMUSP.

2. Professor Titular do Departamento de Obstetrícia e Ginecologia, Disciplina de Obstetrícia, Chefe do Departamento de Obstetrícia e Ginecologia da FMUSP.

Endereço para correspondência: Lílian Renata Fiorelli. Rua Alves Guimarães, 511/64. Jardim América, São Paulo, SP. CEP: 05410-000. email: lilifiorelli@gmail.com 
vivos com peso superior a 4000 gramas é de aproximadamente $9 \%$ com larga variação entre países ${ }^{9}$. No Brasil esta taxa foi de 5\% em 2004 de acordo com os dados do DATASUS ${ }^{19}$, sendo que neste ano a taxa de parto cesáreo em recém-nascidos macrossômicos foi de 49,18\%. No Estado de São Paulo ${ }^{20}$ em 2004, $3,9 \%$ dos recém-nascidos vivos eram macrossômicos, destes, $64,84 \%$ nasceram de parto cesáreo. A preocupação com a prevalência desta entidade na população é embasada no fato que a gestação de fetos macrossômicos está relacionado ao risco aumentado de diversas complicações perinatais ${ }^{5,11,12,14,15,25}$.

A gestação não complicada com suspeita de macrossomia não é uma indicação de primeira cesárea ou indução de parto ${ }^{9}$. Contudo a ocorrência de macrossomia tem sido associada a um aumento na ocorrência de cesáreas ${ }^{6,24}$, além de trauma no parto e morbidade infantil, especialmente quando associada ao diabetes gestacional ${ }^{24}$. As complicações maternas são: trabalho de parto prolongado, atonia uterina, parto operatório, lacerações do trato genital, ruptura uterina e as complicações fetais são: distocia de bisacromial levando a trauma de parto (lesão de plexo braquial, fratura de clavícula) e/ou asfixia $3,4,16,22$.

As definições mais comumente utilizadas referem-se a peso de nascimento acima de 4000 gramas, 4500 gramas ou 4536 gramas ( 10 libras) ${ }^{18,21,23}$. O Colégio Americano de Obstetrícia e Ginecologia considera macrossomia acima de 4500 gramas devido ao aumento significativo da morbidade a partir deste peso, porém reconhece o aumento de risco quando o peso é acima de 4000 gramas $^{2}$.

Considerando estatísticas populacionais, qualquer feto com peso acima do $90^{\circ}$ percentil para a idade gestacional pode ser considerado grande para a idade gestacional. Entretanto o $95^{\circ}$ e o $97,75^{\circ}$ percentil podem ser utilizados como parâmetros pois - $95^{\circ}$ percentil corresponde a 1,96 desvios padrão acima da média e define $90 \%$ da população como peso normal enquanto $97,75^{\circ}$ percentil corresponde a 2 desvios padrão acima da média e define o peso de $95 \%$ da população normal. O percentil de peso para a idade gestacional é considerado melhor para identificar fetos macrossômicos pré-termo ou a termo'.

Em vista da prevalência de macrossomia fetal em nosso país, em especial no estado de São Paulo, suas causas e as complicações inerentes a esta entidade tanto para o recém-nascido como para a mãe, o presente estudo tem como objetivo descrever os resultados perinatais da gestação com feto macrossômico.

\section{METODOLOGIA}

Foram analisados, de modo retrospectivo, 306 partos que ocorreram no Hospital Universitário da Universidade de São Paulo, cujos recém-nascidos apresentaram peso maior ou igual a 4000 gramas no período de 1 de janeiro de 2002 a 31 de dezembro de 2004. Os dados foram obtidos por meio da análise de prontuários.

As características maternas analisadas foram idade, número de gestações, paridade, idade gestacional ao nascimento, tipo de parto e complicações associadas.

Das características do produto conceptual foram analisados o Apgar do primeiro, quinto e décimo minuto, peso e intercorrências.

Os dados foram avaliados por técnicas descritivas e inferenciais por meio de medidas de tendência central, dispersão e correlação (odds ratio ou razão de chances e respectivos intervalos de confiança).

\section{RESULTADOS}

No período de 1 de janeiro de 2002 a 31 de dezembro de 2004 ocorreram 306 partos de recémnascidos com peso igual ou superior a 4000 gramas. As gestantes no presente estudo apresentavam idade entre 14 e 43 anos (27,4 $\pm 6,0$ anos) e destas, 104 (33,9\%) possuíam paridade maior ou igual a dois partos. A idade gestacional variou entre 36 e 43 semanas $(40,1 \pm 1,1)$ sendo $166(54,2 \%)$ gestações pós-data, ou seja, com idade gestacional superior a 40 semanas.

O peso ao nascimento variou de 4000 a 5085 gramas $(4195,5 \pm 188,9)$. O tipo de parto foi via vaginal, normal ou fórcipe, em 141 (46,1\%) gestantes e cesárea em 165 (53,9\%) gestantes.

As principais intercorrências materno-fetais foram 15 casos com mecôneo (4,9\%), 7 casos com traumatismo no recém-nascido (2,3\%), 6 casos com laceração vaginal (1,9\%), 5 casos apresentaram atonia uterina $(1,6 \%)$ e 5 casos que apresentaram distocia do bisacromial (1,6\%). Ocorreram 51 intercorrências materno-fetais (16,6\%). Comparando-se as pacientes submetidas a cesárea e a parto vaginal, não houve diferença significativa em relação a ocorrência de intercorrências $(O R=0,95 ; I C=0,52-1,74)$. Assim como comparando-se recém-nascidos com peso inferior ou superior a 4500 gramas $(O R=1,09$; IC = 0,38-3,10).

\section{DISCUSSÃO}

Os resultados do presente estudo confirmaram a presença de importantes fatores de risco ${ }^{5,23,26}$. A idade materna avançada relacionouse a gestantes com fetos macrossômicos. Outro fator de risco é a multiparidade e foi identificado em 
aproximadamente um terço das gestantes no presente estudo. O pós-datismo foi identificado em mais da metade dos casos estudados.

Mesmo sabendo-se da existência de diversos fatores de risco para a macrossomia, um terço dos casos ainda continuam sem causa definida ${ }^{5,23,26}$; porém, estudos mostram que o risco de macrossomia quase dobra quando existe mais de um fator de risco associado ${ }^{3}$.

Boulet et al. ${ }^{7}$ relatam que a morbidade neonatal aumenta em fetos com peso acima de 4500 gramas, e o risco de mortalidade neonatal aumenta em fetos com peso acima de 5000 gramas $^{7}$. O presente estudo não demonstrou diferença significativa de intercorrências entre gestações cujos recém-nascidos pesaram entre 4000 e 4500 gramas e acima de 4500 gramas. Anoon et al. ${ }^{3}$ estudaram fetos com peso maior ou igual a 5000 gramas observando que as gestantes eram significativamente mais idosas, obesas, multíparas e com diabetes gestacional quando comparadas com gestantes de fetos com peso dentro da normalidade. Além disso, as gestações eram mais prolongadas e a incidência de feto macrossômico em gestações anteriores (o mais importante fator de risco isolado) eram significativamente maiores.

Em nosso estudo mais da metade dos partos foram cesáreas e foi demonstrado que a freqüência de intercorrências materno-fetais não diferiu entre os partos cesáreo ou via vaginal em fetos macrossômicos. A macrossomia é freqüentemente associada a aumento do número de cesáreas ${ }^{3,24}$, contudo, é fun- damental ressaltar que a gestação não complicada com suspeita de macrossomia não é uma indicação de primeira cesárea ou indução de parto ${ }^{9}$. A conduta expectante do trabalho de parto pode ser indicada, pois o diagnóstico pré-natal da macrossomia fetal pode ser impreciso ${ }^{5,23,26}$. Os métodos mais utilizados são o clínico e o ultra-sonográfico ${ }^{23,26}$.

Dentre as complicações maternas identificadas neste estudo a laceração vaginal e a atonia uterina ocorreram em 3,5\% das gestantes levando a hemorragia pós-parto. Kimberly et al. ${ }^{16}$ encontrou a hemorragia pós-parto em ambos os partos vaginal e cesáreo de fetos macrossômicos na mesma proporção. No presente estudo demonstrou-se 1,6\% de distocia de bisacromial. O risco para distocia de ombro na população geral é de aproximadamente $0,9 \%{ }^{22}$ porém, pode ocorrer em $14,6 \%$ dos recém-nascidos com mais de 5000 gramas $^{12}$, podendo causar lesão do plexo braquial ${ }^{3}$ e fratura de clavícula ${ }^{4,22}$.

\section{CONCLUSÃO}

Os fatores de risco para macrossomia, como pós-datismo, multiparidade e idade materna avançada foram identificados no presente estudo. Demonstrouse ainda que, a macrossomia fetal está associada a cesárea, a trauma de parto e a morbidade perinatal, porém, não demonstramos a correlação entre a via de parto ou peso de nascimento acima ou abaixo de 4500 gramas e a freqüência de intercorrências materno-fetais.

Forelli LR, Zugaib M. Perinatal results in fetal macrosomia. Rev Med (São Paulo). 2007 jul.set.;86(3):144-7.

\begin{abstract}
Background: Pregnancy of macrosomic fetus increases risk of several complications. Methods: Retrospective analyse of 306 deliveries occurred in HU/USP between 2002 and 2004 whose newborns had weight $=4000$ gram. Results: Gestational age was between 14 and 43 years $(27,4 \pm 6,0$ years $)$ and $33,9 \%$ with parity $>2$ deliveries. The gestational age varied from 36 to 43 weeks $(40,1 \pm 1,1)$ being $54,2 \%$ postdate gestations, and the born weight was from 4000 to 5085 grames $(4195,5 \pm 188,9)$. Delivery was vaginal in $141(46,1 \%)$ pregnants and cesarean in $165(53,9 \%)$ pregnants. The main maternofetal problems were meconeo $(4,9 \%)$, trauma in newborns $(2,3 \%)$, vaginal laceration $(1,9 \%)$, uterus atonia $(1,6 \%)$ and bisacromial distocia $(1,6 \%)$. There were not significative difference on the occurrence of problems $(\mathrm{OR}=0,95$; $\mathrm{IC}=0,52-1,74)$ between cesarean and vaginal delivery, and between newborns with weight < ou > de 4500 gram $(\mathrm{OR}=1,09 ; \mathrm{IC}=0,38-3,10)$. Conclusion: Postdate, multiparity and advanced maternal age were related with macrosomia. Fetal macrosomia is also related to cesarean, delivery trauma and perinatal morbity, however, it is not show the correlation in delivery way or birthweight $<$ or $>$ of 4500 gram and the frequency of maternofetal problems.
\end{abstract}

KEY WORDS: Fetal macrosomia. Birth weight. Pregnancy complications. Risk factors. 


\section{REFERÊNCIAS}

1. Abramowicz JS, Ahn JT. Fetal macrosomia: diagnosis. Up To Date, 2006. CD-ROM.

2. American College of Obstetricians and Gynecologists. Fetal macrosomia. Practice Bull. 2000; 22.

3. Anoon SS, Rizk DE, Ezimokhai M. Obstetric outcome of excessively overgrown fetuses $(=5000 \mathrm{~g})$ : a casecontrol study. J Perinat Med. 2003;31(4):295-301.

4. Bérard J, Dufour $P$, Vinatier $D$, Subtil $D$, Vanderstichèle S, Monnier JC, Puech F. Fetal macrosomia: risk factors and outcome. A study of the outcome concerning 100 cases < 4500 g. Eur J Obstet Gynecol Reprod Biol. 1998;77:51-9.

5. Berkus MD, Conway D, Langer O. The large fetus. Clin Obstet Gynecol. 1999;42:766-84.

6. Boulet SL, Alexander GR, Salihu HM. Secular trends in cesarean delivery rates among macrosomic deliveries in the United States, 1989 to 2002 . J Perinat Med. 2005;25:569-76.

7. Boulet SL, Birmingham A, Alexander GR, Salihu HM, Pass M. Macrosomic births in the United States: determinants, outcomes, and proposed grades of risk. Am J Obstet Gynecol. 2003;188:1372-8.

8. Catalano P, Kirwan J, Haugel-de Mouzon S, King J. Gestational diabetes and insulin resistance: role in short- and long-term implications for mother and fetus. J Nutr. 2003;133:1674-83.

9. Chauhan SP, Grobman WA, Gherman RA, Chauhan VB, Chang G, Magann EF et al. Suspicion and treatment of the macrosomic fetus: a review. Am J Obstet Gynecol. 2005;193(2):332-46.

10. Chung JH, Voss KJ, Caughey AB, Wing DA, Henderson EJD, Major CA. Role of patient education level in predicting macrosomia among women with gestational diabetes mellitus. J Perinat Med. 2006;26:328-32.

11. Edwards A, Goff J, Baker L. Accuracy and modifying factors of the sonographic estimation of fetal weight in a high-risk population. Aust N Z J Obstet Gynaecol. 2001;41:187-90.

12. Grassi AE, Giuliano MA. The neonate with macrosomia. Clin Obstet Gynecol. 2000;43:340-8.

13. Häger RME, Daltveit AK, Hofoss D, Nilsen ST, Kolaas T, Oian P, et al. Complications of cesarean deliveries: rates and risk factors. Am J Obstet Gynecol.
2004;190:428-34.

14. Haram K, Pirhonen J, Bergsjo P. Suspected big baby: a difficult clinical problem in obstetrics. Acta Obstet Gynecol Scand. 2002;81:185-94.

15. Hellmeyer L, Meyer-Wittkopf M, Ziegler A, Ballast A, Sierra F, Schimidt S. Comparison of sonographic weight estimation to actual BW: a retrospective analysis. Ultraschall Med. 2001;22:167-71.

16. Kimberly DG, Henry OA, Ramicone E, Chan LS, Platt LD. Maternal and infant complications in high and normal weight infants by mode of delivery. Obstet Gynecol. 1998;92:507-13.

17. Langer O. Fetal macrosomia: etiologic factors. Clin Obstet Gynecol. 2000;43:283-97.

18. Menticoglou SM, Manning FA, Morrison I, Harman CR. Must macrosomic fetuses be delivered by a caesarean section? A review of outcome for 786 babies greater than or equal to $4,500 \mathrm{~g}$. Aust N Z J Obstet Gynaecol. 1992;32:100-3.

19. Brasil. Ministério da Saúde. DATASUS. Nascidos vivos - Brasil. Disponível em: http://tabnet.datasus.gov. br/cgi/tabcgi.exe?sinasc/cnv/nvbr.def

20. Brasil. Ministério da Saúde. DATASUS. Nascidos vivos - São Paulo. Disponível em: http://tabnet.datasus. gov. br/cgi/tabcgi.exe?sinasc/cnv/nvSP.def

21. Modanlou HD, Dorchester WL, Thorosian A, Freeman RK. Macrosomia - maternal, fetal and neonatal complications. Obstet Gynecol. 1980;55:420-4.

22. O'Leary JA, Leonetti HB. Shoulder dystocia: prevention and treatment. Am J Obstet Gynecol. 1990;162:5-9.

23. O'Reilly-Green C, Divon M. Sonographic and clinical methods in diagnosis of macrosomia. Clin Obstet Gynecol. 2000;43:309-20.

24. Rodrigues S, Robinson EJ, Kramer MS, Gray-Donald K. High rates of infant macrosomia: a comparison of a Canadian native and a non-native population. J Nutr. 2000;130:806-12.

25. Romoff A. Shoulder dystocia: lessons from the past and emerging concepts. Clin Obstet Gynecol. 2000;43:226-35.

26. Sacks DA, Chenu W. Estimating fetal weight in the management of macrosomia. Obstet Gynecol Surv. 2000;55:229-39. 